\title{
A CIÊNCIA CIDADÃ NA QUESTÃO DOS RESÍDUOS SóLIDOS
}

\section{CITIZEN SCIENCE ON THE SOLID WASTE ISSUE}

Sarita Albagli

Luana Rocha

\begin{abstract}
RESUMO
0 trabalho apresenta resultados de pesquisa sobre a adoção de metodologias e abordagens de ciência cidadã para tratar a questão dos resíduos sólidos. Traça um panorama que contextualiza a problemática dos resíduos sólidos em nível global, sintetiza em seguida os resultados das pesquisas bibliográfica e empírica realizadas e, ao final, apresenta conclusões e recomendações. Foram identificadas 66 iniciativas e experiências, sendo 51 consideradas demonstrativas de distintos focos, públicos-alvos e estratégias de ciência cidadã com essa abordagem, em diferentes países, dentre as quais pouco mais da metade são orientadas para resíduos em ambientes aquáticos, com grande incidência também de iniciativas em resíduos de plástico e microplásticos. A maior parte das iniciativas analisadas é voltada para a produção de dados e informações, contemplando ainda coleta de amostras, monitoramento de impactos, desenvolvimento de soluções e ações coletivas e comunitárias.
\end{abstract}

Palavras-chave: Ciência cidadã. Resíduos sólidos. Desenvolvimento Sustentável. Dados. Informação.

\section{ABSTRACT}

The paper presents research results on the adoption of citizen science methodologies and approaches to address the issue of solid waste. It provides an overview that contextualizes the thematic of solid waste at a global level, then summarizes the results of the bibliographic and empirical research carried out, and, at the end, it presents conclusions and recommendations. The survey identified 66 initiatives and experiences, 51 of which are considered demonstrative of different focuses, target audiences and strategies of citizen science initiatives on solid waste, in different countries, among which slightly more than half are oriented to waste in aquatic environments, with great incidence also for plastic and microplastic waste. Most of the analyzed initiatives are focused on the production of data and information, including sample collection, monitoring of impacts, development of solutions and collective and community actions.

Keywords: Citizen Science. Solid waste. Sustainable development. Data. Information.

Artigo submetido em 29/11/2020 e aceito para publicação em 17/12/2020

1 Pesquisadora do Instituto Brasileiro de Informação em Ciência e Tecnologia, Brasil. Professora do Programa de Pós-Graduação em Ciência da Informação do convênio. Instituto Brasileiro de Informação em Ciência e Tecnologia. Universidade Federal do Rio de Janeiro, Brasil. ORCID https://orcid.org/0000-0003-0030-8964. E-mail: sarita.albagli@gmail.com

2 Assistente de Pesquisa do Instituto Brasileiro de Informação em Ciência e Tecnologia, Brasil. ORCID https://orcid.org/0000-00015602-0375. E-mail: luaroc@gmail.com 


\section{INTRODUÇÃO}

Iniciativas de ciência cidadã vêm se ampliando no mundo, na última década, como forma de aprimorar e promover a contribuição e 0 engajamento de não cientistas nas atividades de produção e análise de dados e informações. Valem-se cada vez mais de ferramentas e tecnologias de informação e comunicação, particularmente plataformas digitais e aplicativos de celular que facilitam a coleta, 0 registro e o compartilhamento desses dados (CONRAD; HILCHEY, 2010; MORESI et al., 2017).

0 conceito de ciência cidadã tem sido entendido e aplicado de diferentes modos, a partir das motivações, questões e situações em que se desenvolve. Em linhas gerais, a ciência cidadã tem se orientado em torno de três grandes eixos (ALBAGLI; ROCHA, 2020):

(a) contribuição voluntária na produção e análise de dados e informações;

(b) divulgação e educação científica;

(c) engajamento cidadão em questões e ações específicas.

Essas iniciativas vêm sendo em grande medida focadas em questões relevantes aos objetivos do desenvolvimento sustentável (ODS) e à transição para uma nova socioeconomia nessas bases.

Dentre essas questões, destaca-se 0 enfrentamento da problemática dos resíduos, em suas diferentes modalidades, ante 0 agravamento da situação do lixo em nível global, observando-se uma crescente demanda pelo desenvolvimento de sistemas de informação orientados para apoiar a tomada de decisão e a busca de soluções nas questões atinentes a esse desafio (KAZA et al. 2018). A gestão efetiva dos resíduos abrange não apenas o descarte, mas também a produção, o consumo, a reciclagem e todos os elementos que envolvem uma economia circular (EUROPEAN PARLIAMENT, 2017), partindo de uma perspectiva de sustentabilidade em âmbito planetário. Isso requer políticas públicas, marcos regulatórios apropriados e, sobretudo, mudanças de comportamento em diferentes níveis -- individual, comunitários, empresarial, governamental.

A ciência cidadã tem-se mostrado uma ferramenta útil para possibilitar modos mais participativos e descentralizados na gestão de resíduos, contribuindo para o envolvimento de diferentes atores, na vizinhança, na localidade e na cidade, e, logo, ampliando a capacidade de coleta, análise e disponibilização de dados nesse tema.

Este trabalho apresenta resultados de pesquisa sobre a adoção de metodologias e abordagens de ciência cidadã para tratar a temática dos resíduos sólidos. Para isso, foi feito um levantamento e 
uma caracterização de iniciativas e experiências demonstrativas de distintos focos, públicos-alvos e estratégias com essa abordagem, em diferentes países. 0 artigo inicia traçando um panorama que contextualiza a questão dos resíduos sólidos em nível global, sintetiza em seguida os resultados da pesquisa realizada e, ao final, apresenta conclusões e recomendações.

\section{DESAFIOS DO SETOR DE RESÍDUOS SÓLIDOS}

0 setor de resíduos abrange a gestão dos seguintes segmentos: os resíduos sólidos urbanos (RSU), os resíduos dos serviços de saúde (RSS), os resíduos sólidos industriais (RSI) e os efluentes líquidos domésticos e industriais.

De acordo com a Política Nacional de Resíduos Sólidos - PNRS $(2010)^{3}$, resíduos sólidos são definidos como:

[...] material, substância, objeto ou bem descartado resultante de atividades humanas em sociedade, a cuja destinação final se procede, se propõe proceder ou se está obrigado a proceder, nos estados sólido ou semissólido, bem como gases contidos em recipientes e líquidos cujas particularidades tornem inviável o seu lançamento na rede pública de esgotos ou em corpos d'água, ou exijam para isso soluções técnica ou economicamente inviáveis em face da melhor tecnologia disponível.

Os resíduos sólidos podem ser também classificados de acordo com a sua origem, tais como estabelecimentos comerciais e prestadores de serviços; residências e limpeza urbana; serviços públicos de saneamento básico; transportes; construção civil; indústria; serviços de saúde; atividades agrossilvopastoris; mineração. Já os resíduos sólidos urbanos são "os resíduos domésticos gerados em áreas urbanas, incluindo os materiais decorrentes de atividades de varrição, limpeza de logradouros, vias públicas e outros serviços de limpeza" (OBSERVATÓRIO DO CLIMA, 2018).

Existe hoje uma verdadeira economia dos resíduos, envolvendo recursos investidos, poupados e gerados; empregos gerados; economia circular (reuso, reciclagem, refabricação, reparo); logística reversa.

Dentre os segmentos do setor de resíduos, o de resíduos sólidos urbanos é onde se observa maior aumento na geração e coleta, com elevação da quantidade de material coletado superior ao

3 Os principais marcos regulatórios de gestão de resíduos sólidos no país são o Plano Nacional de Saneamento Básico (PNSB, previsto na Lei Federal $n^{0} 11.445$ de 2007) e a Política Nacional de Resíduos Sólidos (PNRS, Lei Federal $n^{0}$ 12.305 de 2010). Ambos constituem, por sua vez, desdobramentos de uma série de marcos regulatórios nas áreas ambiental (especialmente no âmbito do Conselho Nacional do Meio Ambiente - Conama) e da saúde pública (especialmente no âmbito da Agência Nacional de Vigilância Sanitária - Avisa), que têm recaído na temática dos resíduos desde a década de 1990. 
aumento da população urbana, pressionando a infraestrutura para sua disposição final (IPEA, 2012). Estima-se que sejam gerados, no mundo, a cada ano, cerca de 1,5 bilhão de toneladas (Gt) de RSU, e que esse volume poderá aumentar para aproximadamente 2,2 Gt até o ano de 2025 (OBSERVATÓRIO DO CLIMA, 2018). Outras estimativas apontam que a produção global de resíduos sólidos urbanos chegará a 11 milhões de toneladas por dia, em 2100 (JOHNSON, s/d).

Dos cerca de 300 milhões de toneladas (Mt) de resíduos gerados no ambiente urbano, em nível mundial, apenas $20 \%$ são reciclados e $13 \%$ são dispostos em aterros sanitários, enquanto cerca de $53 \%$ são destinados a aterros controlados ou lixões, isto é, vazadouros a céu aberto, sem controle do que ali é disposto, e com nenhuma ou reduzidas medidas de proteção ao ambiente (ISWA, 2017; OBSERVATÓRIO DO CLIMA, 2018).

Os lixões continuam a ser usados para destinação de resíduos em praticamente todos os países em desenvolvimento. Ao mesmo tempo, são considerados uma das maiores fontes de poluição do planeta, contaminando 0 ar, os solos e as águas com substâncias tóxicas que afetam a qualidade do ambiente e dos alimentos e, logo, a saúde e a qualidade de vida das pessoas (ISWA, 2017). Frequentemente há pessoas morando e se alimentando nos lixões, bem como catadores de materiais recicláveis, nesses ambientes, sem uso de qualquer proteção (ISWA, 2017).

No Brasil, calcula-se que, em 2018, tenham sido gerados 79 milhões de toneladas de resíduos sólidos urbanos (em média, cerca de $380 \mathrm{~kg} / a n o$ por pessoa, pouco mais de $1 \mathrm{~kg}$ por dia), dos quais 72,7 milhões de toneladas (92\%) foram coletados. A Região Sudeste, onde se concentram $42 \%$ da população, gera cerca da metade dos RSU do país, enquanto a Região Norte é a que apresenta menor percentual de participação no total de RSU coletados (cerca de 6,6\%), mas coleta apenas $81 \%$ dos RSU gerados, mais de $10 \%$ abaixo do percentual médio nacional. É, por outro lado, a região com menor densidade populacional.

Do total de resíduos sólidos urbanos gerados no Brasil, em 2018, 59,5\% tiveram destinação adequada em aterros sanitários. Por outro lado, os demais 40,5\% (quase 30 milhões de toneladas por ano), distribuídos em 3001 municípios brasileiros, foram despejados em locais sem medidas adequadas de proteção à saúde humana e ao meio ambiente -- 23\% em aterros controlados e 17,5\% em lixões (ABRELPE, 2019).

Em 2017, estimou-se a existência no Brasil de cerca de 3000 lixões, o que afetaria 76,5 milhões de pessoas, gerando um gasto anual de mais de $\mathrm{R} \$ 3,6$ bilhões para adoção de medidas visando lidar com os impactos negativos causados no meio ambiente e na saúde pública. Por outro lado, calcula-se 
que a adoção de medidas para dar uma destinação adequada aos resíduos custaria cerca de um terço desse valor (ABRELPE, 2017). Apontam-se como principais barreiras para a erradicação de lixões a pouca disponibilidade de recursos financeiros e a falta de capacitação técnica (ABRELPE, 2015). Nesse aspecto, há também disparidades regionais. Segundo dados da Abrelpe (2019), em 2018, enquanto os estados do Sudeste encaminharam cerca de 49\% de seus RSU para aterros sanitários, na Região Norte, apenas pouco mais de 35\% dos RSU tiveram destinação adequada, sendo quase 35\% ainda depositados em lixões.

0 principal gás produzido pela matéria orgânica contida nos resíduos sólidos, quando depositada em lixões ou aterros, sem contato com o oxigênio livre, é o metano ( $\mathrm{CH} 4)$, que é considerado 28 vezes mais danoso do que o CO2 no agravamento do efeito estufa (ABRELPE, 2013a). Embora a contribuição do setor para o total de emissões em nível nacional tenha sido relativamente baixa, verifica-se que "0 tratamento e disposição final de resíduos sólidos e líquidos podem atingir contribuições percentuais médias de 10\% a 20\% no total de emissões de GEE em diferentes municípios no Brasil” (OBSERVATÓRIO DO CLIMA, 2018).

Ainda de acordo com dados da Abrelpe (2019), observa-se 0 aumento de medidas de coleta seletiva, que já são adotadas por quase três quartos dos municípios brasileiros, embora de modo desigual nos diferentes bairros. Destaca-se o papel dos catadores de materiais reutilizáveis e recicláveis, cuja relevância é reconhecida em diversas partes do mundo, do mesmo modo que sua situação de vulnerabilidade. De acordo com a Aliança Global de Catadores ${ }^{4}$, há cerca de 15 milhões de catadores de lixo informais no mundo, sendo a maioria mulheres, crianças, idosos, migrantes e outros grupos vulneráveis. Em muitos casos, eles são responsáveis por recolher 50 a 100\% do lixo sem custo para as cidades. No Brasil, cooperativas e associações de catadores desempenham um papel crucial na coleta de materiais recicláveis, em todo o país (IPEA, 2015; SILVA 2017).

Uma das consequências do tratamento e da disposição inadequados dos resíduos sólidos urbanos é a poluição de oceanos, sendo que cerca de $80 \%$ do lixo marinho são oriundos de atividades realizadas no continente. Os rios e as cidades costeiras são as principais vias de chegada dos detritos ao mar (JAMBECK et al., 2015; BRASIL, 2019). Dentre os tipos de resíduos que mais poluem os oceanos está o plástico, que pode corresponder a 90\% do volume total, segundo estimativas (BRASIL, 2019). Calcula-se que 8 milhões de toneladas (Mt) de lixo plástico alcancem os mares anualmente (JAMBECK et al., 2015; NAPPER; THOMPSON, 2020). Uma vez no mar, esses detritos se espalham

$4 \quad$ Disponível em: https://globalrec.org/pt-br/ 
rapidamente, pelo deslocamento natural das águas (marés, correntes, ondas etc.), afetando os animais e os ecossistemas marinhos, a economia e a saúde humana (BRASIL, 2019).

0 plástico hoje é considerado uma das maiores ameaças ao meio ambiente, por sua dificuldade de degradação, sua longa permanência no ambiente e sua constante decomposição em partículas cada vez menores, dando origem aos chamados micro e nanoplásticos (BRASIL, 2019; NAPPER; THOMPSON, 2020). No Brasil, o Plano Nacional de Combate ao Lixo no Mar (2019) propõe, dentre outras medidas, de modo articulado com a PNRS, ações de educação e sensibilização sobre a questão da poluição dos oceanos (BRASIL, 2019).

\section{ABORDAGENS PARTICIPATIVAS E CIÊNCIA CIDADÃ}

\subsection{Antecedentes}

A área de resíduos sólidos tem já um significativo acúmulo de experiências participativas, em diferentes partes do mundo (ANEX; FOCHT, 2002). A realização de mutirões e campanhas para coleta de lixo com participação de voluntários, especialmente em áreas costeiras, é prática comum em muitos países.

Em 1986, a ONG Ocean Conservancy lançou a primeira edição da ação International Coastal Cleanup $^{5}$, que em 2020 aconteceu em mais de 100 países. No Brasil, há registros de limpezas comunitárias desde pelo menos a década de 1990, com a mobilização de moradores e associações de bairro, em iniciativas independentes ou em parceria com órgãos da administração pública. A prática do mutirão de limpeza de praias também é comum a várias cidades litorâneas brasileiras, inicialmente com ações mais pontuais, e depois evoluindo para projetos e campanhas encampados por ONGs, empresas e o poder público, com influência de experiências internacionais. Um exemplo é a cartilha "Como fazer um mutirão ecológico", baseada na campanha Clean up the World e publicada pela Secretaria de Meio Ambiente do estado de São Paulo, em 1998 (GOVERNO DO ESTADO DE SÃO PAULO, 1998).

Se, na origem, tais ações estavam mais focadas em realizar trabalho de conscientização da população para o problema do acúmulo de resíduos sólidos e os impactos ambientais causados, ao Iongo do tempo elas foram se transformando e hoje muitas passaram a adotar também metodologias e abordagens de ciência cidadã, como é o caso da própria International Coastal Cleanup.

5 Disponível em: https://oceanconservancy.org/trash-free-seas/international-coastal-cleanup/ 
Organizações atuantes no setor propugnam, cada vez mais, o envolvimento cidadão no desenho e na experimentação de soluções e políticas para a questão (ABRELPE, 2013b; ISWA, 2017; COSTA; PUGLIESI, 2018). Um aspecto considerado importante de envolver as comunidades é que são elas que melhor conhecem o ambiente local, o que é crucial para uma gestão adequada dos resíduos. Abre-se ainda a oportunidade para ampliar a influência de grupos sociais mais marginalizados e vulneráveis, bem como para conectar esforços de pesquisa com interesses e necessidades sociais.

Nesse espírito, a Política Nacional de Resíduos Sólidos (2010) prevê a necessidade de instrumentos de controle social, ali definidos como "conjunto de mecanismos e procedimentos que garantam à sociedade informações e participação nos processos de formulação, implementação e avaliação das políticas públicas relacionadas aos resíduos sólidos". Dentre os princípios da PNRS, ressalta-se "a responsabilidade compartilhada pelo ciclo de vida dos produtos", em que o gerador (seja do lado da produção, seja do consumo) é corresponsável pelos resíduos e sua destinação, reforçando a prática cívica.

\subsection{Iniciativas de ciência cidadã}

Ciência cidadã é uma abordagem relativamente recente para lidar com a questão dos resíduos e do lixo. Ainda que não haja comprovações estatísticas, aponta-se que iniciativas com essa abordagem têm potencialmente contribuições significativas para a produção de dados e informações relevantes à pesquisa científica e à ação governamental, bem como para a conscientização e, logo, um maior engajamento social no enfrentamento do tema. Essas iniciativas vêm crescendo em diferentes países, ante 0 agravamento da situação do lixo, em nível mundial, e a amplificação de seus impactos em diferentes níveis.

0 que principalmente distingue iniciativas de ciências cidadã de outros tipos de iniciativas participativas na questão do lixo e dos resíduos é que as primeiras têm como componente central 0 envolvimento de voluntários e organizações locais na produção, registro e compartilhamento de dados e informações, em grande parte combinados com ações práticas e de educação ambiental, envolvendo também questões como coleta seletiva, reciclagem, compostagem, entre outros.

Neste estudo, foram levantadas e analisadas iniciativas e experiências de ciência cidadã e correlatas, no país e no exterior. 0 objetivo não foi realizar um levantamento extensivo, mas identificar as iniciativas que pudessem servir de exemplos demonstrativos de usos possíveis dessa abordagem no enfrentamento da questão dos resíduos sólidos. 
Nesse levantamento, foram buscadas as seguintes informações:

a) Nome da iniciativa

b) Página web

c) Descrição de objetivos

d) País ou região onde se localiza

e) Vínculo institucional, se houver

f) Recursos técnicos e metodologias utilizados

g) Tipos de dados coletados

h) Informações complementares

Foram levantadas 66 iniciativas participativas relacionadas a lixo e resíduos de diversas naturezas, 51 das quais foram caracterizadas mais propriamente como de ciência cidadã. Destas, 35 (pouco mais da metade) são orientadas para o lixo em ambientes aquáticos (mares, rios, canais, bacias e águas pluviais). A contaminação desses ambientes é um problema crescente, em nível mundial, causada tanto pelo descarte inadequado de resíduos quanto pela deficiência de políticas públicas, sobretudo em regiões e países de menor afluência econômica (como é o caso do Brasil, onde se observa uma parca cobertura da rede de água e esgoto em boa parte dos municípios).

No Brasil, pode-se mencionar como exemplo recente de utilização de metodologias e abordagens de ciência cidadã para lidar com a questão do lixo e dos resíduos no ambiente costeiro, o projeto Recreio Limpo Lab ${ }^{6}$, orientado para promover a qualidade socioambiental do bairro do Recreio dos Bandeirantes, na zona oeste da cidade do Rio de Janeiro, tendo como ponto de partida as praias. Por meio de parceria com a iniciativa Blue Change ${ }^{7}$, foram desenvolvidos dois projetos de ciência cidadã junto com pesquisadoras do Programa de Pós-graduação sobre Dinâmica dos Oceanos e da Terra - UFF, combinando ciências naturais e sociais. A proposta metodológica foi transformar a praia em um laboratório de pesquisa, buscando também compreender a percepção e possíveis mudanças de comportamento dos participantes sobre o lixo na praia.

Observou-se também um número significativo de iniciativas de ciência cidadã orientadas para a temática dos resíduos de plástico e microplástico -- 13 dentre as 51 iniciativas de ciência cidadã analisadas, sendo nove com foco em ambientes aquáticos -, todas surgidas no período de 2010 a 2020

6 Disponível em: https://bluechangeinitiative.wordpress.com/portfolio/recreio-limpo-lab/

7 Disponível em: https://bluechangeinitiative.wordpress.com/ 
(Quadro 1). Os microplásticos são plásticos menores do que $5 \mathrm{~mm}$, incluindo microfibras, derivados de diferentes causas, como exposição ao ambiente, efeitos de correntezas, limpeza de praias e a própria fabricação de objetos, tecidos e roupas com esse material (BRADLEY; STOJKOVIC, 2019). Como já assinalado, a poluição com plásticos é atualmente uma grande preocupação mundial, por seus danos à flora e à fauna dos ambientes aquáticos; à saúde humana, pela contaminação de alimentos; aos sistemas urbanos de escoamento das águas pluviais, entre outros aspectos, o que motiva o engajamento social para a produção de dados e informações, dentre outras ações (ZETTLER et al., 2016; WWF, 2019).

Quadro 1 - Iniciativas focadas em resíduos plásticos

\begin{tabular}{|c|c|c|c|}
\hline Nome/URL & $\begin{array}{l}\text { Abrangência } \\
\text { territorial }\end{array}$ & Atividades/objetivos & Vínculo/Ano de criação \\
\hline $\begin{array}{l}\text { Adventure Scientists' } \\
\text { Global Microplastics } \\
\text { Initiative } \\
\text { https://www. } \\
\text { adventurescientists.org/ } \\
\text { microplastics.html } \\
\end{array}$ & Internacional & $\begin{array}{l}\text { Identificação da extensão da } \\
\text { poluição de microplásticos nos } \\
\text { sistemas marinhos e de água doce } \\
\text { em todo o mundo. }\end{array}$ & $\begin{array}{l}\text { Adventure Scientists } \\
\text { (2013) }\end{array}$ \\
\hline $\begin{array}{l}\text { Earth Challenge } 2020 \\
\text { Plastic Pollution } \\
\text { https:// } \\
\text { earthchallenge2020. } \\
\text { earthday.org/pages/ } \\
\text { plastics }\end{array}$ & Internacional & $\begin{array}{l}\text { Mapeamento dos danos da poluição } \\
\text { global de plástico, tipos e áreas mais } \\
\text { afetadas, por meio de aplicativo } \\
\text { para celular que permite o envio de } \\
\text { fotos e a classificação do material } \\
\text { fotografado. }\end{array}$ & $\begin{array}{l}\text { Earth Day Network } \\
(2020)\end{array}$ \\
\hline $\begin{array}{l}\text { Florida Microplastic } \\
\text { Awareness Project } \\
\text { http://www. } \\
\text { plasticaware.org/ }\end{array}$ & Estados Unidos & $\begin{array}{l}\text { Mapeamento da presença de } \\
\text { microplásticos na costa da Flórida, } \\
\text { por meio de coleta, filtragem } \\
\text { e análise com microscópio de } \\
\text { amostras de água do mar. } \\
\end{array}$ & $\begin{array}{l}\text { University of Florida, } \\
\text { Institute of Food and } \\
\text { Agricultural Sciences } \\
\text { (2015) }\end{array}$ \\
\hline $\begin{array}{l}\text { Investigando la huella } \\
\text { de la basura plástica / } \\
\text { On the track of plastic } \\
\text { waste } \\
\text { https://www.save- } \\
\text { ocean.org/es/ }\end{array}$ & Chile e Alemanha & $\begin{array}{l}\text { Envolvimento de estudantes chilenos } \\
\text { e alemães na coleta e análise de } \\
\text { amostras de lixo e detritos retiradas } \\
\text { de praias. }\end{array}$ & $\begin{array}{l}\text { Kieler } \\
\text { Forschungswerkstatt e } \\
\text { Científicos de la Basura } \\
\text { (2015) }\end{array}$ \\
\hline $\begin{array}{l}\text { Litter-free Digital } \\
\text { Journal } \\
\text { https://www.anecdata. } \\
\text { org/projects/view/122 }\end{array}$ & Estados Unidos & $\begin{array}{l}\text { Rastreamento e remoção de lixos e } \\
\text { detritos de plásticos em ambientes } \\
\text { aquáticos e terrestres. }\end{array}$ & $\begin{array}{l}\text { South Carolina } \\
\text { Aquarium (2016) }\end{array}$ \\
\hline $\begin{array}{l}\text { Marine Debris Tracker } \\
\text { http://marinedebris. } \\
\text { engr.uga.edu/ }\end{array}$ & Internacional & $\begin{array}{l}\text { Aplicativo para rastreamento e } \\
\text { identificação de lixo marinho e } \\
\text { poluição por plásticos no mundo } \\
\text { todo. }\end{array}$ & $\begin{array}{l}\text { Morgan Stanley, } \\
\text { National Geographic } \\
\text { Society, University } \\
\text { of Georgia College of } \\
\text { Engineering (2010) }\end{array}$ \\
\hline
\end{tabular}




\begin{tabular}{|c|c|c|c|}
\hline $\begin{array}{l}\text { Microplastics Citizen } \\
\text { Science Project } \\
\text { https://www. } \\
\text { sustainability.vic.gov. } \\
\text { au/Government/Litter- } \\
\text { and-illegal-dumping/ } \\
\text { Microplastics-Citizen- } \\
\text { Science-Project }\end{array}$ & Austrália & $\begin{array}{l}\text { Registro do lixo visível e coleta } \\
\text { de amostras de areia de praias } \\
\text { locais para posterior análise em } \\
\text { laboratório, contribuindo para } \\
\text { identificação da presença de } \\
\text { microplásticos. }\end{array}$ & $\begin{array}{l}\text { Sustainability Victoria, } \\
\text { EPA Victoria, Melbourne } \\
\text { Water (2017) }\end{array}$ \\
\hline $\begin{array}{l}\text { Nurdle Patrol } \\
\text { www.nurdlepatrol.org }\end{array}$ & Estados Unidos & $\begin{array}{l}\text { Identificação, mapeamento e } \\
\text { remoçãa de nurdles (pequenas } \\
\text { pastilhas de plástico usadas na } \\
\text { fabricação de produtos de plástico) } \\
\text { em áreas costeiras. }\end{array}$ & $\begin{array}{l}\text { Mission-Aransas } \\
\text { National Estuarine } \\
\text { Research Reserve, } \\
\text { University of Texas } \\
\text { Marine Science Institute } \\
\text { (2018) }\end{array}$ \\
\hline $\begin{array}{l}\text { OpenLitterMap.com } \\
\text { https://openlittermap. } \\
\text { com/ }\end{array}$ & Internacional & $\begin{array}{l}\text { Aplicativo para mapeamento da } \\
\text { poluição por plástico em todo } 0 \\
\text { mundo. }\end{array}$ & $\begin{array}{l}\text { Organização própria } \\
\text { (2015) }\end{array}$ \\
\hline $\begin{array}{l}\text { Plastic Pirates } \\
\text { https://bmbf-plastik.de/ } \\
\text { en/plasticpirates }\end{array}$ & Alemanha & $\begin{array}{l}\text { Iniciativa voltada para jovens de } \\
10 \text { a } 16 \text { anos visando identificar } \\
\text { a presença de plásticos em rios. } \\
\text { Integra o estudo "Plastics in the } \\
\text { Environment", do Ministério de } \\
\text { Educação e Pesquisa da Alemanha. }\end{array}$ & $\begin{array}{l}\text { German Federal } \\
\text { Ministry of Education } \\
\text { and Research (BMBF) } \\
(2016)\end{array}$ \\
\hline $\begin{array}{l}\text { Plastic Spotter } \\
\text { https://plasticspotter.nl/ }\end{array}$ & Holanda & $\begin{array}{l}\text { Identificação e mapeamento da } \\
\text { presença de plástico em canais da } \\
\text { cidade de Leiden, com mutirões } \\
\text { de limpeza com uso de canoas e } \\
\text { caiaques. Envio dos dados coletados } \\
\text { por meio do aplicativo CrowdWater. }\end{array}$ & $\begin{array}{l}\text { Leiden University } \\
\text { (2019) }\end{array}$ \\
\hline $\begin{array}{l}\text { The Big Microplastic } \\
\text { Survey } \\
\text { https:// } \\
\text { microplasticsurvey.org/ } \\
\text { about-the-project }\end{array}$ & Internacional & $\begin{array}{l}\text { Identificação da presença de } \\
\text { microplásticos por meio da coleta } \\
\text { e análise de amostras de areia e } \\
\text { de água de rios, lagos e regiões } \\
\text { costeiras. }\end{array}$ & $\begin{array}{l}\text { Just One Ocean and } \\
\text { the University of } \\
\text { Portsmouth (2018) }\end{array}$ \\
\hline $\begin{array}{l}\text { The Plastic Tide } \\
\text { https://www. } \\
\text { theplastictide.com/ }\end{array}$ & Internacional & $\begin{array}{l}\text { Classificação online de fotos } \\
\text { de plástico e lixo marinho para } \\
\text { treinamento de um algoritmo de } \\
\text { detecção automática de poluição em } \\
\text { imagens registradas por drones. }\end{array}$ & $\begin{array}{l}\text { Organização própria } \\
\text { (2016) }\end{array}$ \\
\hline
\end{tabular}

Fonte: Elaboração própria

\subsection{Focos e ferramentas}

Iniciativas participativas e de ciência cidadã têm-se voltado para diferentes etapas do ciclo de vida dos resíduos sólidos, incluindo geração, coleta e disposição / destinação (aterros sanitários, aterros controlados, lixões, incineração). Tipos de dados variados têm sido contemplados, como: tipos de resíduos, fontes e proveniência, quantidades, localização, incidência temporal e evolução. 
No levantamento realizado, observou-se que os objetivos e resultados almejados são diversos, tais como:

- $\quad$ mobilização e capacitação dos cidadãos para produzirem e utilizarem dados e ampliarem 0 conhecimento sobre padrões e tipos de lixo gerado e seus impactos no meio ambiente e na saúde humana (por exemplo, o Bay Drift ${ }^{8}$ nos Estados Unidos);

- $\quad$ subsídio à proposição de ações sobre essas questões, contribuindo para o desenvolvimento de alternativas sustentáveis para a gestão do lixo, considerando os pontos de vista das populações locais e grupos afetados (como o estudo participativo Cumulative Environmental Effects: Expanding Research with the Hopi Tribe ${ }^{9}$, nos Estados Unidos);

- $\quad$ conscientização em nível local sobre o problema global do lixo, combatendo a chamada "cegueira do lixo" (trash blindness), ou seja, quando as pessoas não se dão conta nem compreendem problemas relacionados à má disposição do lixo na natureza e em suas comunidades (por exemplo, iniciativas que promovem ações de limpeza de costas e outras localidades, como a Litter-free Digital Journal ${ }^{10}$, nos Estados Unidos, e a Map It for World Cleanup Day ${ }^{11}$, baseada na Estônia e de abrangência internacional).

As iniciativas de ciência cidadã podem focar grupos específicos, como estudantes, professores e escolas (como a Científicos de la Basura, no Chile), mergulhadores e ativistas na questão do lixo e outras agendas socioambientais locais (como a Dive Against Debris ${ }^{12}$, nos Estados Unidos). Isso varia também segundo cada contexto. Por exemplo, estudos verificaram que projetos costeiros participativos atraem um público mais diverso, pela facilidade de acesso ao local, do que os desenvolvidos em ambientes marinhos em alto mar, que envolvem públicos mais especializados, como os praticantes de pesca, mergulho e outros esportes aquáticos (MILACH et al., 2017).

São cada vez mais importantes as questões ambientais e sanitárias envolvidas na questão dos resíduos, como poluição do ar, do solo e das águas, doenças e questões de saúde pública (como propagação de vetores de enfermidades); a prevenção e consequências de desastres advindos da sua má gestão, como enchentes e deslizamentos; e os efeitos sobre a mudança climática (gases de efeito

8 Disponível em: https://scistarter.org/bay-drift

9 Disponível em: https://ciehr.arizona.edu/content/cumulative-environmental-effects-expanding-research-hopi-tribe

10 Disponível em: https://www.anecdata.org/projects/view/122

11 Disponível em: https://www.worldcleanupday.org/map-it

12 Disponível em: https://www.projectaware.org/diveagainstdebris 
estufa gerados). Essas questões variam segundo as especificidades dos diferentes tipos de resíduos, suas origens e características dos ambientes em que são produzidos e dispostos.

A maior parte das iniciativas de ciência cidadã na área de resíduos sólidos, analisadas neste estudo, envolve a contribuição de voluntários na produção, registro e análise de dados e informações que estabeleçam linhas de base, permitam o monitoramento de mudanças e a projeção de tendências, contribuindo para políticas públicas e mudanças de comportamento social e empresarial e subsidiando pesquisas científicas. Aí incluem-se dados sobre proveniência, tipos e quantidades de resíduos, bem como locais mais frequentemente encontrados e variações ao longo do tempo, ajudando a identificar os produtos que mais comumente se transformam em lixo e, logo, fontes de poluição. Como exemplos, podem ser citadas as iniciativas: Mutirão de Limpeza do Plano Nacional de Combate ao Lixo no Mar ${ }^{13}$, no Brasil; Cleaner Canals Glasgow ${ }^{14}$, na Escócia; Australian Marine Debris Initiative - AMDI ${ }^{15}$, na Austrália; Morro Bay Trash Tracker ${ }^{16}$, nos Estados Unidos; China Coastline Monitoring and Cleanup ${ }^{17}$, na China. Há ainda iniciativas que se orientam para:

- $\quad$ coleta de amostras de materiais com presença de resíduos, como de areia da praia, e registro de lixo visível (por exemplo, a Microplastics Citizen Science Project ${ }^{18}$, na Austrália);

- $\quad$ mapeamento e medição de impactos e outros problemas associados ao lixo, como mau cheiro (por exemplo, a D-NOSES ${ }^{19}$, no âmbito da União Europeia; e a COASST Marine Debris Project ${ }^{20}$, nos Estados Unidos);

- $\quad$ mapeamento e notificação de áreas com lixo disposto irregularmente, seja na vizinhança, seja em ambientes silvestres (como a TrashOut ${ }^{21}$, na Estônia);

- desenvolvimento participativo de soluções a respeito da disposição de resíduos, boa parte dos quais em ambientes costeiros, marinhos e fluviais, bem como de alternativas sustentáveis à queima e despejo irregular de resíduos (por exemplo, a Global Alliance for Incinerator Alternatives - GAIA ${ }^{22}$;

13 Disponível em: https://www.gov.br/mma/pt-br/assuntos/agendaambientalurbana/combate-ao-lixo-no-mar

14 Disponível em: https://www.scottishcanals.co.uk/news/children-and-community-volunteers-become-citizen-scientists-to-tackle-litter-in-glasgow-and-west-dunbartonshire/

15 Disponível em: https://www.tangaroablue.org /

16 Disponível em: https://www.mbnep.org/trash-tracker/

17 Disponível em: https://www.globalgiving.org/projects/china-coastline-monitoring-and-cleanup/

18 Disponível em: https://www.sustainability.vic.gov.au/Government/Litter-and-illegal-dumping/Microplastics-Citizen-Science-Project

19 Disponível em: https://dnoses.eu/

20 Disponível em: https://coasst.org/about/our-story/

21 Disponível em: https://www.trashout.ngo/

22 Disponível em: https://www.no-burn.org/ 
- classificação de imagens para treinamento de inteligência artificial e algoritmos desenvolvidos para dar suporte à localização de resíduos em áreas extensas e de difícil acesso, como oceano (por exemplo, The Plastic Tide ${ }^{23}$, na Inglaterra)

Junto com a coleta e o registro de dados, têm-se o envolvimento e 0 apoio das comunidades em ações de limpeza e coleta (como a Love your Coast ${ }^{24}$, na Nova Zelândia).

Há também registro de iniciativas de monitoramento participativo e voluntário de poluentes orgânicos persistentes (persistent organic pollutants - POPs), que têm uma natureza bioacumulativa e efeitos adversos em organismos marinhos e pessoas (como a International Pellet Watch ${ }^{25}$ - IPW, baseado no Japão).

Dentre as metodologias e ferramentas adotadas para essas iniciativas de ciência cidadã, destacam-se:

- $\quad$ a utilização de cartões de dados para preenchimento pelos voluntários (como 0 Great Canadian Shoreline Cleanup ${ }^{26}$, no Canadá);

- $\quad$ a disponibilização de aplicativos de celular para facilitar o mapeamento, o registro, 0 envio, 0 compartilhamento e a (geo)visualização de dados para download e uso dos participantes (como o Marine Debris Tracker27, o Beach Observer ${ }^{28}$, Canadá, e o Clean Swell ${ }^{29}$, Estados Unidos);

- 0 desenvolvimento de plataformas online interativas, visando facilitar redes de aprendizado e desenvolvimentos coletivos em cocriação (por exemplo, a World Waste Platform da Let's Do It Foundation ${ }^{30}$, Estônia; e 0 Marine LitterWatch ${ }^{31}$, no âmbito da União Europeia);

- $\quad$ a estruturação de bases de dados de modo a facilitar o registro, a recuperação, a disponibilização e a análise dos dados, coletados pelos voluntários, sobre a produção e coleta de lixo (por exemplo, a Litter Intelligence ${ }^{32}$, Nova Zelândia), transformados em dados públicos com possibilidade de

23 Disponível em: https://www.theplastictide.com/

24 Disponível em: https://sustainablecoastlines.org/about/our-programmes/love-your-coast/

25 Disponível em: http://pelletwatch.org/

26 Disponível em: https://www.shorelinecleanup.ca/

27 Disponível em: http://marinedebris.engr.uga.edu/

28 Disponível em: https://scistarter.org/beachobserver. Cabe ressaltar que esse aplicativo não está mais disponível para uso.

29 Disponível em: https://oceanconservancy.org/trash-free-seas/international-coastal-cleanup/cleanswell/

30 Disponível em: https://opendata.letsdoitworld.org/\#l

31 Disponível em: https://www.eea.europa.eu/themes/water/europes-seas-and-coasts/assessments/marine-litterwatch\#tab-news-and-articles

32 Disponível em: $\underline{\text { https://litterintelligence.org/ }}$ 
acesso a relatórios para download (como o Ocean Conservancy TIDES Database ${ }^{33}$ e 0 Marine Debris Monitoring and Assessment Project - MDMAP34, ambos nos Estados Unidos);

- $\quad 0$ treinamento dos voluntários para coleta de dados, de modo adequado a seus usos científicos (como a já mencionada Litter Intelligence, Nova Zelândia);

- $\quad$ a produção coletiva e a utilização de marcadores visuais, como cartões de sinalização (drift cards), com a participação de voluntários e ativistas, indicando locais de maior incidência de resíduos e a necessidade de ações de coleta e de iniciativas educacionais preventivas (como na Bay Drift, nos Estados Unidos);

- $\quad$ outros meios, como o uso de caiaques e canoas para observação e a coleta de lixo nos rios (por exemplo, o RiverSweep ${ }^{35}$, Estados Unidos), bem como a participação e 0 treinamento de mergulhadores na identificação, avaliação de impacto e, eventualmente, coleta de lixo marinho (como 0 Dive Against Debris, nos Estados Unidos);

- $\quad$ e ainda o uso de metodologias como pesquisa participativa baseada na comunidade (communitybased participatory research - CBPR) e ferramentas de pesquisa e inovação responsáveis ( $R R I$ tools).

\subsection{Questões e desafios}

Iniciativas e projetos de ciência cidadã enfrentam, de modo geral, alguns desafios, além daqueles mais específicos às questões a que se dirigem e aos contextos em que se desenvolvem.

Um primeiro ponto a ressaltar é que não se trata de processos rápidos de implementação. Estes exigem identificar e estabelecer canais de comunicação com possíveis participantes e colaboradores, desenvolvendo estratégias para seu envolvimento desde a fase preparatória do projeto, bem como para reter seus interesses e manter esse engajamento ao longo do desenvolvimento do projeto e seu eventual desdobramento posterior. Frequentemente observa-se a pouca sensibilização por parte de segmentos envolvidos e afetados na problemática enfocada, em âmbitos social, governamental e privado/empresarial.

Estratégias de sensibilização, de comunicação e de educação -- seja para buscar adesões, seja para divulgar e dar retorno à população e demais grupos interessados sobre resultados da iniciativa e

33 Disponível em: https://www.coastalcleanupdata.org/

34 Disponível em: https://marinedebris.noaa.gov/research/marine-debris-monitoring-and-assessment-project

35 Disponível em: https://scistarter.org/riversweep 
seus possíveis usos -- precisam ir além dos meios tradicionais de comunicação científica (encontros e periódicos científicos) e mesmo de divulgação e popularização da ciência. Isso requer também definir que aspectos e questões da temática tratada -- neste caso, a gestão de resíduos sólidos -- podem mobilizar e contar com a contribuição de não cientistas ou especialistas.

A comunicação é vista aqui não apenas como uma atividade informativa ou motivadora, mas, sobretudo, como "escuta, diálogo e aprendizado" (ABRELPE, s/d), ressaltando-se que:

No caso particular de transformações no comportamento de geração e descarte de resíduos, mais ainda, por se tratar de uma prática que acontece diariamente, em ambiente privado e ligada a escolhas íntimas e de consumo, logo, com identidade e expressão (ABRELPE, s/d).

Outro aspecto diz respeito ao estabelecimento e 0 acesso a infraestruturas condizentes para coletar, registrar e fazer uso dos dados, para desenvolver e testar protocolos, ferramentas e formatos de participação e contribuição cidadã, havendo ainda as barreiras no acesso à internet enfrentadas por muitos grupos sociais e regiões. Isso envolve também, em alguns casos, oferecer treinamento para o uso dessas infraestruturas e metodologias, seja na contribuição com dados e informações, seja no aproveitamento de seus resultados.

Cabe ainda considerar as questões específicas de cada área ou região, como no caso de áreas e comunidades de difícil acesso. Na questão do lixo, essas áreas e comunidades são particularmente afetadas pela deficiência na coleta e disposição dos resíduos. Um exemplo é o problema do acúmulo de lixo em aldeias indígenas e outras comunidades tradicionais, localizadas muitas vezes em áreas remotas, gerado tanto pelo consumo dos moradores, como trazido por visitantes, incluindo produtos embalados em plástico, pilhas, entre outros. Nessas comunidades, que não contam com serviços de saneamento básico, coleta seletiva e outros serviços públicos e informações adequadas às suas realidades, os resíduos sólidos são frequentemente queimados, contribuindo para a emissão de gases de efeito estufa e para incêndios florestais, ou são enterrados, contribuindo para a poluição do solo e da água e pondo em risco a vida animal.

Nesse caso, temos no Brasil, embora não se caracterize propriamente como projeto de ciência cidadã, porque não envolve produção e compartilhamento de dados, a iniciativa Aldeia Lixo Zero ${ }^{36}$ (Zero Waste Community), que trabalha com famílias que vivem em áreas isoladas na Amazônia brasileira e com lideranças de comunidades tradicionais para conscientizar sobre os perigos do descarte e queima de resíduos na floresta, além de governos locais e estaduais e cooperativas para melhorar a destinação

36 Disponível em: https://aldeialixozero.org/ 
e a reciclagem de resíduos sólidos. 0 projeto desenvolve ações como: capacitação, inclusive para compostagem de resíduos orgânicos; organização de limpeza comunitária; remoção de resíduos sólidos para reciclagem; construção de estações de reciclagem de resíduos nas comunidades; suprimento de baterias recarregáveis; provisão de contêineres para diferentes tipos de resíduos; colocação de sinais de lixo zero para orientar visitantes; apoio à implementação das recomendações da PNRS e à institucionalização de cooperativas de catadores locais.

Além dessas questões, há os aspectos trazidos pela pandemia da covid-19. A realização de iniciativas de ciência cidadã nesse contexto, no mundo todo, tem enfrentado dois tipos de repercussão. Por um lado, iniciativas que envolvem atividades presenciais e observações em campo são dificultadas pelos limites à mobilidade física e à proximidade social impostos pela doença (ROSE; SURI; BROOKS; RYAN, 2020), afetando particularmente iniciativas voltadas para o tema dos resíduos sólidos. Por outro, contribuições pela internet são incentivadas e favorecidas, seja porque as pessoas permanecem conectadas online por mais tempo, seja porque se tornam mais familiarizadas com o uso de mídias digitais (KORNFELD, 2020). Além disso, há mobilizações e iniciativas cidadãs ou com participação cidadã que surgem para lidar com a situação da pandemia e para desenvolver ações colaborativas e de apoio mútuo. (BOWSER; PARKER; LONG, 2020; CLINIO et al. 2020).

\section{CONSIDERAÇÕES FINAIS}

Observa-se então que o desenvolvimento de iniciativas de ciência cidadã em geral, e mais particularmente no apoio à gestão sustentável de resíduos, envolve diferentes dimensões: (a) socioculturais, como disponibilidade e condições de adesão às iniciativas; (b) político-institucionais, como existência de organizações públicas, privadas e não governamentais que participem ou apoiem a iniciativa, na sua implementação e manutenção, bem como no uso dos dados e resultados alcançados; (c) econômicas, como condições financeiras para desenvolver e manter as iniciativas e infraestruturas pertinentes; (d) técnicas, como capacidade para desenvolver e disponibilizar aplicativos, páginas webs, bases de dados e outros dispositivos e sistemas necessários ao desenvolvimento das iniciativas, bem como disponibilidade de infraestrutura prévia, incluindo conexão à internet; (e) científicas, como conhecimento e disposição para orientar e utilizar os resultados alcançados.

Essas são questões que afetam uma boa governança e a responsabilidade compartilhada entre os vários atores na questão dos resíduos sólidos, sendo aspectos cruciais na gestão efetiva dos resíduos. 


\section{AGRADECIMENTOS}

Este estudo é parte do Projeto "Pesquisa Analítica para a Gestão Sustentável dos Resíduos Sólidos na Amazônia Legal", desenvolvido no âmbito da Coordenação de Tecnologias Aplicadas a Novos Produtos - COTEA do Instituto Brasileiro de Informação em Ciência e Tecnologia - IBICT.

\section{REFERÊNCIAS}

ALBAGLI, S.; ROCHA, L. Ciência cidadã no Brasil: um estudo exploratório. In: BORGES, M.M.; CASADO, E.S. (Orgs.). Sob a lente da Ciência aberta: olhares de Portugal, Espanha e Brasil. Coimbra: Universidade de Coimbra, 2020. p 489-511.

ANEX, R.P.; FOCHT, W. Public participation in Life Cycle Assessment and Risk Assessment: a shared need. Risk Analysis, v. 22, n. 5, 2002.

ABRELPE. Associação Brasileira de Empresas de Limpeza Pública e Resíduos. Manual de comunicação social e engajamento para gestão de resíduos. Abrelpe; Climate and Clean Air Coalition Municipal Solid Waste Initiative (CCAC MSWI), s/l, s/d.

ABRELPE. Associação Brasileira de Empresas de Limpeza Pública e Resíduos. Atlas Brasileiro de Emissões de GEE e Potencial Energético na Destinação de Resíduos Sólidos. São Paulo: Abrelpe, 2013a.

ABRELPE. Associação Brasileira de Empresas de Limpeza Pública e Resíduos. Resíduos sólidos: manual de boas práticas de planejamento. São Paulo: Abrelpe; ISWA, 2013b. Disponível em: https:// smastr16.blob.core.windows.net/cpla/2017/04/manual-boas-praticas.pdf Acesso em: 17 dez. 2020.

ABRELPE. ASSOCIAÇÃO BRASILEIRA DE EMPRESAS DE LIMPEZA PÚBLICA E RESÍDUOS. Panorama de Resíduos Sólidos no Brasil 2015. São Paulo: Abrelpe, 2015. Disponível em: https:// abrelpe.org.br/panorama/ . Acesso em 18 dez 2020.

ABRELPE. ASSOCIAÇÃO BRASILEIRA DE EMPRESAS DE LIMPEZA PÚBLICA E RESÍDUOS. Panorama de Resíduos Sólidos no Brasil 2017. São Paulo: Abrelpe, 2017. Disponível em: https:// abrelpe.org.br/panorama/. Acesso em 18 dez 2020.

ABRELPE. Associação Brasileira de Empresas de Limpeza Pública e Resíduos. Panorama de Resíduos Sólidos no Brasil 2018/2019. São Paulo: Abrelpe: São Paulo, 2019. Disponível em: https:// abrelpe.org.br/panorama/ . Acesso em 18 dez 2020.

BRADLEY, C.; STOJKOVIC, S. Microplastics and Port Phillip Bay. Summary report. Sustainability Victoria: Melbourne, 2019

BRASIL. MINISTÉRIO DO MEIO AMBIENTE. Agenda Nacional de Qualidade Ambiental Urbana: plano de combate ao lixo no mar. Brasília: Ministério do Meio Ambiente, 2019. 41 p. 
BOWSER, A.; PARKER, A.; LONG, AI Citizen Science and COVID-19: The Power of the (Distanced) Crowd. Ctrl Forward: blog of the Science and Technology Innovation Program at the Wilson Center. Washington. 22 jun. 2020. Disponível em: https://www.wilsoncenter.org/blog-post/citizen-scienceand-covid-19-power-distanced-crowd. Acesso em: 17 dez. 2020.

CLINIO, A.; MARTINS, B.C.; METZKER NETTO, C.; TAVARES, L.E.; OLIVEIRA, M.L.S.; GUIMARÃES, R.M.S. Segura a Onda: experiência brasileira de mapeamento de iniciativas cidadãs no enfrentamento da pandemia da Covid-19. Liinc em Revista, v. 16, n.2, Dezembro de 2020.

CONRAD, C. C.; HILCHEY, K. G.. A review of citizen science and community-based environmental monitoring: issues and opportunities. Environmental Monitoring and Assessment, 176 (1-4), 273291, 2010.

COSTA, A. M.; PUGLIESI, E. Análise dos manuais para elaboração de planos municipais de gestão integrada de resíduos sólidos. Eng Sanit Ambient, v.23 n.3, maio/jun 2018, p. 509-516

EUROPEAN PARLIAMENT. Towards a circular economy - Waste management in the EU. EPRS | European Parliamentary Research Service. Scientific Foresight Unit (STOA). PE 581.913Study IP/G/ STOA/FWC/2013-001/LOT 3/C3. September 2017. Disponível em: https://www.europarl.europa.eu/ RegData/etudes/STUD/2017/581913/EPRS_STU(2017)581913_EN.pdf . Acesso em 18 dez 2020.

GOVERNO DO ESTADO DE SÃO PAULO, Secretaria do Meio Ambiente, Coordenadoria de Educação Ambiental - CEAM; Instituto Ecoar para a Cidadania. Como fazer um mutirão ecológico: Campanha "clean up the world" vamos limpar o mundo. São Paulo, 1998. Disponível em: https://www. infraestruturameioambiente.sp.gov.br/educacaoambiental/prateleira-ambiental/como-fazer-ummutirao-ecologico-campanha-clean-up-the-world-vamos-limpar-0-mundo-2/. Acesso em: 15 de dez. 2020.

IPEA. Boas Práticas de Gestão de Resíduos Sólidos Urbanos e de Logística Reversa com a Inclusão de Catadoras e de Catadores de Materiais Recicláveis. Rio de Janeiro: IPEA, 2015.

IPEA. Diagnóstico dos Resíduos Sólidos Urbanos: Relatório de Pesquisa. Brasília: IPEA, 2012.

ISWA. Associação Internacional de Resíduos Sólidos. Roteiro para o encerramento de lixões: os lugares mais poluídos do mundo. São Paulo: Abrelpe: 2017. Disponível em: http://abrelpe.org.br/ pdfs/publicacoes/roteiro-para-encerramento-de-lixoes.pdf Acesso em: 18 dez 2020.

ISWA. Associação Internacional de Resíduos Sólidos. Prevenção ao lixo marinho - agora! Uma parceria facilitada pela ISWA para prevenir o lixo marinho, com um chamado global para a ação para investir na gestão sustentável de resíduos e recursos em todo o mundo. São Paulo: Abrelpe, 2018. Disponível em: https://abrelpe.org.br/prevencao-a-poluicao-marinha/ Acesso em 18 dez 2020.

JAMBECK, J. R.; GEYER, R.; WILCOX, C.; SIEGLER, T. R.; PERRYMAN, M.; ANDRADY, A.; NARAYAN, R.; LAW, K. L.. Plastic waste inputs from land into the ocean. Science, [S.L.], v. 347, n. 6223, p. 768771, 12 fev. 2015. American Association for the Advancement of Science (AAAS). Disponível em: http://dx.doi.org/10.1126/science.1260352. Acesso em 18 dez 2020.

JOHNSON, N. Urban Waste Analytics Using data analytics and citizen science to improve waste understanding and operations. Warwick Institute for the Science of Cities. The University of Warwick. 
s/d. Disponível em: https://wisc.warwick.ac.uk/files/6414/6222/1818/Nick.pdf . Acesso em 18 dez 2020.

KAZA, Silpa; YA0, Lisa C.; BHADA-TATA, Perinaz; VAN WOERDEN, Frank. 2018. What a Waste 2.0: A Global Snapshot of Solid Waste Management to 2050. Urban Development;. Washington, DC: World Bank Group, 2018. Disponível em: https://openknowledge.worldbank.org/handle/10986/30317 . Acesso em 17 dez 2020.

KORNFELD, Maddie. As Scientists Struggle with Rollbacks, Stay At Home Orders and Funding Cuts, Citizens Fill the Gap. Inside Climate News. Nova York, 23 jun. 2020. Disponível em: https:// insideclimatenews.org/news/23062020/citizen-science-coronavirus/. Acesso em: 17 dez. 2020.

MILACH, S., ESTEVES, M. G. P., BARROS, S. R. S., MEIRIÑO, M. J., SOUZA, J. M. Ciência cidadã como instrumento de suporte à gestão de ambientes marinhos e costeiros. Anais do $\mathbf{X}$ Encontro Nacional de Gerenciamento Costeiro - X ENCOGERCO CIDEC Sul. Rio Grande: FURG, 2017. p. 305306

MORESI, E. A. D.; BARBOSA, J.A.; BRAGA FILHO, M.O.; ALVES, P.N.; SANTOS, J.C.A. ASSIS, F.C.S.; BERNARDES, T.M., Lima, V.C. 0 emprego do aplicativo Sci-Hub em projetos de ciência cidadã.

Revista Iberoamericana de Sistemas, Cibernética e Informática, 14 (2), 45-52. 2017. Recuperado em 29 de janeiro de 2019, de: http://www.iiisci.org/Journal/riSCI/FullText.asp?var=\&id=CA301DY17

NAPPER, Imogen Ellen; THOMPSON, Richard C.. Plastic Debris in the Marine Environment: history and future challenges. Global Challenges, [S.L.], v. 4, n. 6, 6 abr. 2020. Wiley. Disponível em: http:// dx.doi.org/10.1002/gch2.201900081. Acesso em: 17 dez. 2020.

OBSERVATÓRIO DO CLIMA. Emissões de GEE no Brasil e suas implicações para políticas públicas e a contribuição brasileira para o Acordo de Paris. Documento de Análise. Período 1970 - 2016. Observatório do Clima, 2018.

ROSE, Sanjo; SURI, Jessleena; BROOKS, Michael; RYAN, Peter G. COVID-19 and citizen science: lessons learned from Southern Africa. Ostrich, [S.L.], v. 91, n. 2, p. 188-191, 2 abr. Disponível em: 2020. National Inquiry Services Center (NISC). http://dx.doi.org/10.2989/00306525.2020.1783589. Acesso em: 17 dez. 2020.

SILVA, Sandro Pereira. A organização coletiva de catadores de material reciclável no Brasil: dilemas e potencialidades sob a ótica da economia solidária. Brasília: IPEA, 2017.

WWF. Solucionar a poluição plástica: transparência e responsabilização. Brasília: WWF, 2019.

ZETTLER, E., TAKADA, H., MONTELEONE, B., MALLOS, N., ERIKSEN, M.; AMARAL-ZETTLER, L. Incorporating citizen science to study plastics in the environment. Analytical Methods, n.9, 2017, p. 1392-1403. 